\title{
Properties of third-order nonlinear differential equations
}

\section{Blanka Baculíková and Jozef Džurina*}

\author{
"Correspondence: \\ jozef.dzurina@tuke.sk \\ Department of Mathematics, \\ Faculty of Electrical Engineering and \\ Informatics, Technical University of \\ Košice, Letná 9, 04200 Košice, \\ Slovakia
}

\begin{abstract}
We establish new comparison theorems, to offer criteria for all nonoscillatory solutions of the third-order functional differential equation$$
\left[r(t)\left[x^{\prime}(t)\right]^{\gamma}\right]^{\prime \prime}+p(t) x^{\beta}(\tau(t))=0
$$

tend to zero. We consider both delay and advanced case of studied equation. The results obtained essentially improve and complement earlier ones.

MSC: $34 \mathrm{~K} 11 ; 34 \mathrm{C} 10$

Keywords: third-order functional differential equations; comparison theorem; oscillation
\end{abstract}

\section{Introduction}

We are concerned with the asymptotic behavior of all solutions of the third-order functional differential equations

$$
\left[r(t)\left[x^{\prime}(t)\right]^{\gamma}\right]^{\prime \prime}+p(t) x^{\beta}(\tau(t))=0 .
$$

Throughout the article, we will assume $r, p \in C\left(\left[t_{0}, \infty\right)\right), \tau \in C^{1}\left(\left[t_{0}, \infty\right)\right)$ and

(H1) $\gamma, \beta$ are the ratios of two positive odd integers,

(H2) $r(t)>0, p(t)>0, \tau^{\prime}(t)>0, \lim _{t \rightarrow \infty} \tau(t)=\infty$.

In the sequel, it is assumed that $(E)$ is in a canonical form, i.e.,

$$
R(t)=\int_{t_{0}}^{t} r^{-1 / \gamma}(s) \mathrm{d} s \rightarrow \infty \quad \text { as } t \rightarrow \infty
$$

By a solution of Equation $(E)$ we mean a function $x(t) \in C^{1}\left(\left[T_{x}, \infty\right)\right), T_{x} \geq t_{0}$, which has the property $r(t)\left(x^{\prime}(t)\right)^{\gamma} \in C^{2}\left(\left[T_{x}, \infty\right)\right)$ and satisfies Equation $(E)$ on $\left[T_{x}, \infty\right)$. We consider only those solutions $x(t)$ of $(E)$ which satisfy $\sup \{|x(t)|: t \geq T\}>0$ for all $T \geq T_{x}$. We assume that $(E)$ possesses such a solution. A solution of $(E)$ is called oscillatory if it has arbitrarily large zeros on $\left[T_{x}, \infty\right)$ and otherwise it is called to be nonoscillatory.

Various techniques were established for examination of $(E)$ and its particular cases. In the articles [1-4], the authors have introduced comparison theorems for comparing studied equation with a set of the first order delay/advanced equation, in the sense that oscillation of these first order equations yields desired properties of third order equation. 
Properties of $(E)$ have been usually studied under condition $\beta=\gamma$ or $\beta<\gamma$. In this article we establish results, where $\beta \geq \gamma$. Moreover, in the cited articles the authors in generally consider either delay or advanced equations, but our technique permits to study both advanced and delayed cases. On the other hand, in the existing comparison results of this kind, there are studied equations always compared with canonical second order differential equation, but in this article we were able to establish comparison with noncanonical differential equation.

We offer new comparison principles, in which we compare our third order equation with the second order differential inequality and this reduction essentially simplifies the investigation of the properties of third order differential equations. Our results generalize those presented in [1-16].

Remark 1.1 All functional inequalities considered in this article are assumed to hold eventually, that is, they are satisfied for all $t$ large enough.

\section{Main results}

In the following lemma, we present the classification of the possible nonoscillatory solutions of $(E)$.

Lemma 2.1 Let $x(t)$ be a nonoscillatory solution of $(E)$. Then $x(t)$ satisfies, one of the following conditions:

$$
\begin{aligned}
& \left(C_{1}\right) \quad x(t) x^{\prime}(t)<0, \quad x(t)\left[r(t)\left[x^{\prime}(t)\right]^{\gamma}\right]^{\prime}>0, \quad x(t)\left[r(t)\left[x^{\prime}(t)\right]^{\gamma}\right]^{\prime \prime}<0, \\
& \left(C_{2}\right) \quad x(t) x^{\prime}(t)>0, \quad x(t)\left[r(t)\left[x^{\prime}(t)\right]^{\gamma}\right]^{\prime}>0, \quad x(t)\left[r(t)\left[x^{\prime}(t)\right]^{\gamma}\right]^{\prime \prime}<0,
\end{aligned}
$$

eventually.

Proof The proof follows immediately from the canonical form of $(E)$ and details are left to a reader.

To simplify our formulations of the main results, we recall the following definition:

Definition 2.1 We say that $(E)$ enjoys property (A) if every its nonoscillatory solution satisfies $\left(C_{1}\right)$.

Remark 2.1 It is easy to verify that condition

$$
\int_{t_{0}}^{\infty} p(s) \mathrm{d} s=\infty
$$

guarantees property $(\mathrm{A})$ of $(E)$. Consequently, in the sequel, we may assume that the integral on the left hand side of (2.1) is convergent.

Property (A) of $(E)$ has been studied by various authors (see enclosed references). We offer new technique for investigation property $(\mathrm{A})$ of $(E)$ based on the comparison theorems, in which we reduce property $(\mathrm{A})$ of $(E)$ to the absence of certain positive solution of the suitable second order differential inequality. At first, we establish criteria for property (A) of advanced differential equation. We start with the following auxiliary result. 
Lemma 2.2 Let $\tau(t) \geq t$. Assume that $x(t)$ satisfies $\left(C_{2}\right)$. Then for any $k \in(0,1)$

$$
|x(\tau(t))| \geq k \frac{R(\tau(t))}{R(t)}|x(t)|
$$

eventually.

Proof Assume that $x(t)>0$. The monotonicity of $w(t)=r(t)\left[x^{\prime}(t)\right]^{\gamma}$ implies that

$$
\begin{aligned}
x(\tau(t))-x(t) & =\int_{t}^{\tau(t)} x^{\prime}(s) \mathrm{d} s=\int_{t}^{\tau(t)} w^{1 / \gamma}(s) r^{-1 / \gamma}(s) \mathrm{d} s \\
& \geq w^{1 / \gamma}(t) \int_{t}^{\tau(t)} r^{-1 / \gamma}(s) \mathrm{d} s=w^{1 / \gamma}(t)[R(\tau(t))-R(t)] .
\end{aligned}
$$

That is,

$$
\frac{x(\tau(t))}{x(t)} \geq 1+\frac{w^{1 / \gamma}(t)}{x(t)}[R(\tau(t))-R(t)]
$$

On the other hand, since $x(t) \rightarrow \infty$ as $t \rightarrow \infty$, then for any $k \in(0,1)$ there exists a $t_{1}$ large enough, such that

$$
k x(t) \leq x(t)-x\left(t_{1}\right)=\int_{t_{1}}^{t} w^{1 / \gamma}(s) r^{-1 / \gamma}(s) \mathrm{d} s \leq w^{1 / \gamma}(t) \int_{t_{1}}^{t} r^{-1 / \gamma}(s) \mathrm{d} s \leq w^{1 / \gamma}(t) R(t)
$$

or equivalently,

$$
\frac{w^{1 / \gamma}(t)}{x(t)} \geq \frac{k}{R(t)} .
$$

Using (2.4) in (2.3), we get

$$
\frac{x(\tau(t))}{x(t)} \geq 1+\frac{k}{R(t)}[R(\tau(t))-R(t)] \geq k \frac{R(\tau(t))}{R(t)} .
$$

This completes the proof.

Let us denote

$$
p_{1}(t)=\frac{R^{\beta}(\tau(t))}{R^{\beta}(t)} p(t) .
$$

Theorem 2.1 Let $\tau(t) \geq t$. If for some $c \in(0,1)$ the second order differential inequality

$$
\left(\frac{1}{p_{1}^{1 / \beta}(t)}\left(z^{\prime}(t)\right)^{1 / \beta}\right)^{\prime}+c \frac{t^{1 / \gamma}}{r^{1 / \gamma}(t)} z^{1 / \gamma}(t) \leq 0
$$

has not any solution satisfying

$$
z(t)>0, \quad z^{\prime}(t)<0, \quad\left(\frac{1}{p_{1}^{1 / \beta}(t)}\left(z^{\prime}(t)\right)^{1 / \beta}\right)^{\prime}<0,
$$

then (E) has property (A). 
Proof Assume the contrary, let $x(t)$ be a nonoscillatory solution of Equation $(E)$, satisfying $\left(C_{2}\right)$. We may assume that $x(t)>0$, for $t \geq t_{0}$. Setting (2.2) into $(E)$, we obtain

$$
\left[r(t)\left[x^{\prime}(t)\right]^{\gamma}\right]^{\prime \prime}+k^{\beta} p(t) \frac{R^{\beta}(\tau(t))}{R^{\beta}(t)} x^{\beta}(t) \leq 0
$$

On the other hand, it follows from the monotonicity of $y(t)=\left[r(t)\left[x^{\prime}(t)\right]^{\gamma}\right]^{\prime}$, that

$$
r(t)\left[x^{\prime}(t)\right]^{\gamma} \geq \int_{t_{1}}^{t} y(s) \mathrm{d} s \geq y(t)\left(t-t_{1}\right) \geq c_{1}^{\gamma / \beta} t y(t)
$$

eventually, where $c_{1} \in(0,1)$ is an arbitrary chosen constant. Evaluating $x^{\prime}(t)$ and then integrating from $t_{1}\left(\geq t_{0}\right)$ to $t$, we are lead to

$$
x(t) \geq c_{1}^{1 / \beta} \int_{t_{1}}^{t} \frac{s^{1 / \gamma}}{r^{1 / \gamma}(s)} y^{1 / \gamma}(s) \mathrm{d} s
$$

Setting to (2.6), we have

$$
y^{\prime}(t)+c_{1} k^{\beta} p_{1}(t)\left[\int_{t_{1}}^{t} \frac{s^{1 / \gamma}}{r^{1 / \gamma}(s)}\left(\left[r(s)\left[x^{\prime}(s)\right]^{\gamma}\right]^{\prime}\right)^{1 / \gamma} \mathrm{d} s\right]^{\beta} \leq 0
$$

Integrating from $t$ to $\infty$, one gets

$$
y(t) \geq c \int_{t}^{\infty} p_{1}(s)\left[\int_{t_{1}}^{s} \frac{u^{1 / \gamma}}{r^{1 / \gamma}(u)} y^{1 / \gamma}(u) \mathrm{d} u\right]^{\beta} \mathrm{d} s
$$

where $c=c_{1} k^{\beta}$. Let us denote the right hand side of (2.9) by $z(t)$. Then $y(t) \geq z(t)>0$ and $z(t)$ satisfies $\left(P_{1}\right)$ and moreover,

$$
\begin{aligned}
0 & =\left(\frac{1}{p_{1}^{1 / \beta}(t)}\left(z^{\prime}(t)\right)^{1 / \beta}\right)^{\prime}+c \frac{t^{1 / \gamma}(t)}{r^{1 / \gamma}(t)} y^{1 / \gamma}(t) \\
& \geq\left(\frac{1}{p_{1}^{1 / \beta}(t)}\left(z^{\prime}(t)\right)^{1 / \beta}\right)^{\prime}+c \frac{t^{1 / \gamma}(t)}{r^{1 / \gamma}(t)} z^{1 / \gamma}(t) .
\end{aligned}
$$

Consequently, $z(t)$ is a solution of the differential inequality $\left(E_{1}\right)$, which contradicts our assumption.

We are prepared to establish the corresponding result also for delay differential equations. Let us denote

$$
p_{2}(t)=\frac{p\left(\tau^{-1}(t)\right)}{\tau^{\prime}\left(\tau^{-1}(t)\right)}
$$

Theorem 2.2 Let $\tau(t) \leq t$. Iffor some $c \in(0,1)$ the second order differential inequality

$$
\left(\frac{1}{p_{2}^{1 / \beta}(t)}\left(z^{\prime}(t)\right)^{1 / \beta}\right)^{\prime}+c \frac{t^{1 / \gamma}}{r^{1 / \gamma}(t)} z^{1 / \gamma}(t) \leq 0
$$


has not any solution satisfying

$$
z(t)>0, \quad z^{\prime}(t)<0, \quad\left(\frac{1}{p_{2}^{1 / \beta}(t)}\left(z^{\prime}(t)\right)^{1 / \beta}\right)^{\prime}<0
$$

then (E) has property (A).

Proof Assume the contrary, let $x(t)$ be a positive solution of Equation $(E)$, satisfying $\left(C_{2}\right)$. An integration of $(E)$ from $t$ to $\infty$, yields

$$
\begin{aligned}
{\left[r(t)\left[x^{\prime}(t)\right]^{\gamma}\right]^{\prime} } & \geq \int_{t}^{\infty} p(s) x^{\beta}(\tau(s)) \mathrm{d} s=\int_{\tau(t)}^{\infty} \frac{p\left(\tau^{-1}(s)\right)}{\tau^{\prime}\left(\tau^{-1}(s)\right)} x^{\beta}(s) \mathrm{d} s \\
& \geq \int_{t}^{\infty} \frac{p\left(\tau^{-1}(s)\right)}{\tau^{\prime}\left(\tau^{-1}(s)\right)} x^{\beta}(s) \mathrm{d} s .
\end{aligned}
$$

Using (2.8), one can see that $y(t)=\left[r(t)\left[x^{\prime}(t)\right]^{\gamma}\right]^{\prime}$ satisfies

$$
y(t) \geq c_{1} \int_{t}^{\infty} p_{2}(s)\left[\int_{t_{1}}^{s} \frac{u^{1 / \gamma}}{r^{1 / \gamma}(u)} y^{1 / \gamma}(u) \mathrm{d} u\right]^{\beta} \mathrm{d} s .
$$

Let us denote the right hand side of (2.11) by $z(t)$. Then similarly as in the proof of Theorem 2.1, we can verify that $z(t)$ is a positive solution of $\left(E_{2}\right)$ and moreover, it satisfies $\left(P_{2}\right)$, which contradicts our assumption.

Establishing, new criteria for elimination of solutions of $\left(E_{i}\right)$ satisfying $\left(P_{i}\right), i=1,2$, we immediately obtain sufficient conditions for property $(\mathrm{A})$ of $(E)$. Since $\left(E_{1}\right)$ and $\left(E_{2}\right)$ are of the same form, we present just one general criterion and then, we adapt them for both $\left(E_{i}\right)$. So we consider the noncanonical differential inequality

$$
\left(a(t)\left(z^{\prime}(t)\right)^{\alpha}\right)^{\prime}+b(t) z^{\delta}(t) \leq 0
$$

where

(H3) $\alpha, \delta$ are the ratios of two positive odd integers,

(H4) $a(t)>0, b(t)>0$.

Let us denote

$$
\varrho(t)=\int_{t}^{\infty} a^{-1 / \alpha}(s) \mathrm{d} s
$$

Theorem 2.3 Assume that $\delta>\alpha$. Iffor all $k>0$

$$
\limsup _{t \rightarrow \infty} \int_{t_{0}}^{t}\left[\varrho^{\delta}(s) b(s)-\frac{\delta \alpha^{\alpha}}{k(\alpha+1)^{\alpha+1} \varrho(s) a^{1 / \alpha}(s)}\right] \mathrm{d} s>\frac{1}{k},
$$

then (E") has not any solution satisfying

$$
z(t)>0, \quad z^{\prime}(t)<0, \quad\left(a(t)\left(z^{\prime}(t)\right)^{\alpha}\right)^{\prime}<0 .
$$


Proof Let $z(t)$ be a positive solution of $\left(E^{*}\right)$, such that $\left(P^{*}\right)$ holds. We define

$$
w(t)=\frac{a(t)\left(z^{\prime}(t)\right)^{\alpha}}{z^{\delta}(t)} .
$$

Then $w(t)<0$ and, moreover,

$$
w^{\prime}(t)=\frac{\left(a(t)\left(z^{\prime}(t)\right)^{\alpha}\right)^{\prime}}{z^{\delta}(t)}-\delta w(t) \frac{z^{\prime}(t)}{z(t)} \leq-b(t)-\delta w^{1+1 / \alpha}(t) \frac{z^{-1+\delta / \alpha}(t)}{a^{1 / \alpha}(t)} .
$$

On the other hand, noting that $-\left(a(t)\left(z^{\prime}(t)\right)^{\alpha}\right)^{1 / \alpha}$ is positive and increasing, we see that there exists a constant $k_{1}>0$ such that $-\left(a(t)\left(z^{\prime}(t)\right)^{\alpha}\right)^{1 / \alpha} \geq k_{1}$ and

$$
\begin{aligned}
z(t) & \geq \int_{t}^{\infty}-z^{\prime}(s) \mathrm{d} s=\int_{t}^{\infty}-\left(a(s)\left(z^{\prime}(s)\right)^{\alpha}\right)^{1 / \alpha} a^{-1 / \alpha}(s) \mathrm{d} s \\
& \geq-\left(a(t)\left(z^{\prime}(t)\right)^{\alpha}\right)^{1 / \alpha} \varrho(t) \geq k_{1} \varrho(t),
\end{aligned}
$$

or equivalently

$$
z^{-1+\delta / \alpha}(t) \geq k_{2} \varrho^{-1+\delta / \alpha}(t)
$$

where $k_{2}=k_{1}^{-1+\delta / \alpha}$. Setting (2.15) into (2.13), one gets

$$
w^{\prime}(t) \leq-b(t)-\delta k_{2} w^{1+1 / \alpha}(t) \frac{\varrho^{-1+\delta / \alpha}(t)}{a^{1 / \alpha}(t)} .
$$

It is useful to notice that (2.14) implies

$$
z^{\alpha-\delta}(t) \geq-w(t) \varrho^{\alpha}(t)
$$

which together with (2.15) implies

$$
\frac{1}{k_{2}^{\alpha}} \geq-w(t) \varrho^{\delta}(t)
$$

Multiplying (2.16) by $\varrho^{\delta}(t)$ and then integrating from $t_{1}$ to $t$, we are lead to

$$
\begin{aligned}
& w(t) \varrho^{\delta}(t)-w\left(t_{1}\right) \varrho^{\delta}\left(t_{1}\right)+\delta \int_{t_{1}}^{t} \frac{\varrho^{\delta-1}(s)}{a^{1 / \alpha}(s)} w(s) \mathrm{d} s \\
& \quad \leq-\int_{t_{1}}^{t} b(s) \varrho^{\delta}(s) \mathrm{d} s-\delta k_{2} \int_{t_{1}}^{t} \frac{\varrho^{\delta-1+\delta / \alpha}(s)}{a^{1 / \alpha}(s)} w^{1+1 / \alpha}(s) \mathrm{d} s,
\end{aligned}
$$

which in view of (2.17) yields

$$
\int_{t_{1}}^{t}\left\{b(s) \varrho^{\delta}(s)+\delta\left[\frac{\varrho^{\delta-1}(s)}{a^{1 / \alpha}(s)}\left(w(s)+k_{2} \varrho^{\delta / \alpha}(s) w^{1+1 / \alpha}(s)\right)\right]\right\} \mathrm{d} s \leq \frac{1}{k_{2}^{\alpha}} .
$$

An elementary calculation shows that for the function $f(u)=u+A u^{1+1 / \alpha}, u<0$ the following estimate holds

$$
u+A u^{1+1 / \alpha} \geq-\frac{\alpha^{\alpha}}{(\alpha+1)^{\alpha+1}} \frac{1}{A^{\alpha}} .
$$


Consequently,

$$
w(s)+k_{2} \varrho^{\delta / \alpha}(s) w^{1+1 / \alpha}(s) \geq-\frac{\alpha^{\alpha}}{(\alpha+1)^{\alpha+1}} \frac{1}{k_{2}^{\alpha} \varrho^{\delta}(s)} .
$$

Therefore,

$$
\int_{t_{1}}^{t}\left[\varrho^{\delta}(s) b(s)-\frac{\delta \alpha^{\alpha}}{k(\alpha+1)^{\alpha+1} \varrho(s) a^{1 / \alpha}(s)}\right] \mathrm{d} s \leq \frac{1}{k},
$$

with $k=k_{2}^{\alpha}$. Taking $\lim \sup _{t \rightarrow \infty}$ on both sides, we get a contradiction with (2.12). This finishes our proof.

Now, we transform condition (2.12) to the more practical form.

Corollary 2.1 Assume that $\delta>\alpha$. If

$$
\lim _{t \rightarrow \infty} \varrho^{\delta+1}(t) b(t) a^{1 / \alpha}(t)=\infty
$$

then $\left(E^{*}\right)$ has not any solution satisfying $\left(P^{*}\right)$.

Proof It follows from (2.18) that for any $k>0$

$$
\varrho^{\delta+1}(t) b(t) a^{1 / \alpha}(t) \geq \frac{\delta \alpha^{\alpha}}{k(\alpha+1)^{\alpha+1}}+\frac{1}{k},
$$

eventually. That is

$$
\varrho^{\delta}(t) b(t)-\frac{\delta \alpha^{\alpha}}{k(\alpha+1)^{\alpha+1} \varrho(t) a^{1 / \alpha}(t)} \geq \frac{1}{k} \frac{1}{\varrho(t) a^{1 / \alpha}(t)} .
$$

Integrating the above inequality from $t_{1}$ to $t$, one gets

$$
\int_{t_{1}}^{t} \varrho^{\delta}(s)\left\{b(s)-\frac{\delta \alpha^{\alpha}}{k(\alpha+1)^{\alpha+1} \varrho(s) a^{1 / \alpha}(s)}\right\} \mathrm{d} s=\frac{1}{k}\left(\ln \frac{1}{\varrho(t)}-\ln \frac{1}{\varrho\left(t_{1}\right)}\right) .
$$

Letting $t \rightarrow \infty$, we see that (2.12) holds true and the assertion now follows from Theorem 2.3

We combine Theorems 2.1 and 2.2 together with Corollary 2.1 , to obtain easily verifiable criteria for property $(\mathrm{A})$ of $(E)$.

Theorem 2.4 Let $\beta>\gamma, \tau(t) \geq t$. If

$$
\lim _{t \rightarrow \infty}\left(\int_{t}^{\infty} \frac{R^{\beta}(\tau(s))}{R^{\beta}(s)} p(s) \mathrm{d} s\right)^{1+1 / \gamma} \frac{t^{1 / \gamma}}{r^{1 / \gamma}(t)} \frac{R^{\beta}(t)}{p(t) R^{\beta}(\tau(t))}=\infty,
$$

then $(E)$ has property $(A)$. 
Proof We set $\alpha=1 / \beta, \delta=1 / \gamma, a(t)=p_{1}^{-1 / \beta}(t)$, and $b(t)=c \frac{t^{1 / \gamma}}{r^{1 / \gamma}(t)}$. Then $\varrho(t)=\int_{t}^{\infty} p_{1}(s) \mathrm{d} s$. Since (2.18) reduces to (2.19), Corollary 2.1 ensures that $\left(E_{1}\right)$ has not any solution satisfying $\left(P_{1}\right)$. The assertion now follows from Theorem 2.1.

Theorem 2.5 Let $\beta>\gamma, \tau(t) \leq t$. If

$$
\lim _{t \rightarrow \infty}\left(\int_{t}^{\infty} p(s) \mathrm{d} s\right)^{1+1 / \gamma} \frac{\tau^{1 / \gamma}(t)}{r^{1 / \gamma}(\tau(t))} \frac{\tau^{\prime}(t)}{p(t)}=\infty
$$

then (E) has property (A).

Proof We set $\alpha=1 / \beta, \delta=1 / \gamma, a(t)=p_{2}^{-1 / \beta}(t)$, and $b(t)=c \frac{t^{1 / \gamma}}{r^{1 / \gamma}(t)}$. Then $\varrho(t)=\int_{\tau^{-1}(t)}^{\infty} p(s) \mathrm{d} s$. As (2.18) reduces to (2.20), Corollary 2.1 guarantees that $\left(E_{2}\right)$ has not any solution satisfying $\left(P_{2}\right)$. The assertion now follows from Theorem 2.2.

Remark 2.2 For $\tau(t) \equiv t$ both conditions (2.19) and (2.20) simplifies to the same condition

$$
\lim _{t \rightarrow \infty}\left(\int_{t}^{\infty} p(s) \mathrm{d} s\right)^{1+1 / \gamma} \frac{t^{1 / \gamma}}{r^{1 / \gamma}(t)} \frac{1}{p(t)}=\infty
$$

for property (A) of $(E)$.

Corollary 2.2 Assume that (E) enjoys property (A). If moreover,

$$
\int_{t_{0}}^{\infty} \frac{1}{r^{1 / \gamma}(v)}\left(\int_{v}^{\infty} \int_{u}^{\infty} p(s) \mathrm{d} s \mathrm{~d} u\right)^{1 / \gamma} \mathrm{d} v=\infty
$$

then every nonoscillatory solution of $(E)$ tends to zero as $t \rightarrow \infty$.

Proof Since $(E)$ has property (A), every its nonoscillatory solution satisfies $\left(C_{1}\right)$, and what is more, (2.21) ensures that such solution tends to zero as $t \rightarrow \infty$.

Example 2.1 Consider the third order nonlinear differential equation

$$
\left(t\left(x^{\prime}(t)\right)^{3}\right)^{\prime \prime}+\frac{a}{t^{2}} x^{5}(\lambda t)=0, \quad t \geq 1,
$$

where $a>0$ and $\lambda>0$. Since both conditions (2.19) and (2.20) hold, Theorems 2.4 and 2.5 imply that $\left(E_{x 1}\right)$ enjoys property $(\mathrm{A})$ and, moreover, Corollary 2.2 guarantees that every nonoscillatory solution of $\left(E_{x 1}\right)$ tends to zero as $t \rightarrow \infty$. For $a=30 \lambda^{5}$ one such solution is $x(t)=1 / t$.

\section{Summary}

Our results can be applied to both delay and advanced third order differential equations. The criteria obtained are easy verifiable and have been precedented by suitable joint illustrative example.

Our method essentially simplifies the examination of the third order equations and what is more, it supports backward the research on the second order delay/advanced differential equations and inequalities. 


\section{Competing interests}

The authors declare that they have no competing interests.

\section{Authors' contributions}

The authors have made the same contribution. All authors read and approved the final manuscript.

\section{Acknowledgement}

This work was supported by the Slovak Research and Development Agency under the contract No. APVV-0008-10.

Received: 18 January 2012 Accepted: 22 June 2012 Published: 10 July 2012

\section{References}

1. Agarwal, RP, Grace, SR, O'Regan, D: On the oscillation of certain functional differential equations via comparison methods. J. Math. Anal. Appl. 286, 577-600 (2003) Zbl 1057.34072

2. Agarwal, RP, Grace, SR, Smith, T: Oscillation of certain third order functional differential equations. Adv. Math. Sci. Appl. 16, 69-94 (2006) Zbl 1116.34050

3. Baculíková, B, Džurina, J: Oscillation of third-order neutral differential equations. Math. Comput. Model. 52, 215-226 (2010) Zbl 1201.34097

4. Grace, SR, Agarwal, RP, Pavani, R, Thandapani, E: On the oscillation of certain third order nonlinear functional differential equations. Appl. Math. Comput. 202, 102-112 (2008) Zbl 1154.34368

5. Baculíková, B, Džurina, J: Oscillation of third-order nonlinear differential equations. Appl. Math. Lett. 24, 466-470 (2011) Zbl 1209.34042

6. Baculíková, B: Properties of third order nonlinear functional differential equations with mixed arguments. Abstr. Appl. Anal. 2011, 1-15 (2011) Zbl 1217.34109

7. Baculíková, B, Džurina, J: Oscillation of third-order functional differential equations. Electron. J. Qual. Theory Differ. Equ. 43, 1-10 (2010) Zbl 1211.34077

8. Cecchi, M, Došlá, Z, Marini, M: On third order differential equations with property A and B. J. Math. Anal. Appl. 231(2), 509-525 (1999) Zbl 0926.34025

9. Džurina, J: Asymptotic properties of third order delay differential equations. Czechoslov. Math. J. 45, 443-448 (1995) Zbl 0842.34073

10. Džurina, J: Comparison theorems for functional differential equations with advanced argument. Boll. Unione Mat Ital., A 7, 461-470 (1993) Zbl 0803.34066

11. Erbe, LH, Kong, Q, Zhang, BG: Oscillation Theory for Functional Differential Equations. Marcel Dekker, New York (1994) Zbl 0821.34067

12. Györi, I, Ladas, G: Oscillation Theory of Delay with Applications. Clarendon Press, Oxford (1991) Zbl 0780.34048

13. Kusano, T, Naito, M: Comparison theorems for functional differential equations with deviating arguments. J. Math. Soc. Jpn. 3, 509-533 (1981) Zbl 0494.34049

14. Ladde, GS, Lakshmikantham, V, Zhang, BG: Oscillation Theory of Differential Equations with Deviating Arguments. Marcel Dekker, New York (1987) Zbl 0832.34071

15. Parhi, N, Padhi, S: On oscillation and asymptotic property of a class of third-order differential equations. Czechoslov. Math. J. 49(1), 21-33 (1999) Zbl 0955.34023

16. Philos, ChG: On the existence of nonoscillatory solutions tending to zero at $\infty$ for differential equations with positive delay. Arch. Math. 36, 168-178 (1981) Zbl 0463.34050

doi:10.1186/1687-1847-2012-103

Cite this article as: Baculíková and Džurina: Properties of third-order nonlinear differential equations. Advances in Difference Equations 2012 2012:103.

\section{Submit your manuscript to a SpringerOpen ${ }^{\circ}$ journal and benefit from:}

- Convenient online submission

- Rigorous peer review

- Immediate publication on acceptance

Open access: articles freely available online

- High visibility within the field

- Retaining the copyright to your article 